\title{
Primordial Black Hole Formation from Inflaton
}

\author{
Xin He MENG ${ }^{1}$, Bin WANG ${ }^{2}$ and S.Feng ${ }^{3}$ \\ CCAST B.O.Box 8370, Beijing \\ 1.Departments of Physics, Nankai University \\ Tianjin, P.R.China \\ Department of Physics, Lancaster University, UK \\ E-mail: mengxh@public1.tpt.tj.cn
}

\author{
2.Department of Physics, Shanghai Normal University, P.R.China \\ 3.Department of Physics, University of Science and Technology, P.R.China
}

\begin{abstract}
Measurements of the distances to SNe Ia have produced strong evidence that the Universe is really accelarating, implying the existence of a nearly uniform component of dark energy with the simplest explanation as a cosmological constant. In this paper a small changing cosmological term is proposed, which is a function of a slow-rolling scalar field, by which the de Sitter primordial black holes' properties, for both charged and uncharged cases, are carefully examined and the relationship between the black hole formation and the energy transfer of the inflaton within this cosmological term is eluciated.
\end{abstract}

There is now prima facie evidence that supports two basic tenets, inflation and dark (matter and energy) components of the hot big bang universe paradign 1 . Measurements of the distances to SNe Ia have produced strong evidence that the Universe is indeed accelarating which indicates that most of the critical density exists in the form of nearly uniform and positive dark energy. This component is poorly understood. So, naturally the identification and elucidation of the mysterious dark-energy component is a very pressing question for nowadays physics. Vacuum energy is only the simplest possibility for the smooth dark component; there are other possibilitied: frustrated topological defects or a slow rolling scalar field or quintessence. Independent evidence for the existence of this dark energy, e.g., by CMB anisotropy, the SDSS and $2 \mathrm{dF}$ surveys, or gravitational lensing, is crucial for verifying the accounting of matter and energy in the Universe. Additional and more precious measurements of SNe Ia could help shed light on the precise nature of the dark energy. The dark energy problem is not only of great importance for cosmology, but also for fundamental physics as well. Whether it is vacuum energy or quintessence, it is still a puzzle for fundamental physics and possibly a clue about the unification of the forces and particle 14 .

For the not very clear dark matter identity, Primordial black holes (PBHs) are also one of the possible cold dark matter candidates which are believed to take the majority of the matter contents of the Universe. PBHs may form 
in the early universe when pre-existing adiabatic density fluctuations enter into the cosmological horizon and recollapse. That is, primordial overdensities seeded, for instance by inflation, may collapse to primordial black holes during early eras if they exceed a critical threshold . Thus, it is quite reasonable to discuss PBHs by connecting the mysterious dark energy problems to PBHs' formation in the de Sitter spacetimes where a cosmological term is essential to describe the PBHs properties.

In this paper we propose a tiny changing cosmological term dependent on a slow-rolling scalar field, which may come from a supersymmetric particle physics model at higher energy scale, just as some classes of quintessence which may originate from the dynamic supersymmetry breaking of a supersymmetry particle theory with a flat direction 15 . We discuss its relation with de-Sitter primordial black holes formation for both charged and uncharged cases, as well as the black holes's properties.

Studies of black hole formation from gravitational collapse of a (massless) scalar field have revealed interesting nonperturbative and non-linear energy (mass) converting phenomena at the threshold of black hole formation 17 . Specifically, starting from the spherically symmetrical de Sitter black hole spacetimes with charges $\mathrm{q}$ and mass $\mathrm{m}$,

$$
d s^{2}=-a(t, r) d t^{2}+a^{-1}(t, r) d r^{2}+r^{2} d \Omega^{2},
$$

where $d \Omega \equiv d \theta^{2}+\sin ^{2} \theta d \varphi^{2}$, and $\left\{x^{\mu}\right\}=\{t, r, \theta, \varphi\}$ are the usual spherical coordinates,

$$
a(t, r)=1-2 m / r-\Lambda \times r^{2} / 3+q^{2} / r^{2}
$$

The $\Lambda$ is taken the similar form given by Peebles and Vilenkin 18 with a notation that now we only consider a single component field case for simplicity and the reduced Planck mass is set $M_{p}=(8 \pi G)^{-1 / 2}=1$, as well as $c=\hbar=1$

$$
\Lambda(\phi)=b\left(\phi^{4}+M^{4}\right)
$$

where the constant energy parameter is assumed to dominate and the selfcoupling constant $b=1 \times 10^{-14}$ from the condition that present-day large scale structure grows from quantum fluctuations frozen into $\phi$ during inflation 15.13 as well as the considerations that the present density parameter in matter is $\Omega_{m} \approx 0.3$, with $\Omega_{\phi}=1-\Omega_{m} \approx 0.7$ in the inflaton $\mathrm{B}_{3}$. Besides, we also require the cosmological term satisfy the flatness conditions at the very early Universe evolution stage to ensure its slow changing property,

$$
\dot{\phi} \approx-\Lambda(\phi)^{\prime} / H
$$


where $\mathrm{H}$ is the Hubble parameter which is a function of the inflaton $\phi$ for the very early Universe evolution period, the overhead dot referring to derivative to time and the prime indicating derivative with respect to the inflaton $\phi$. The two flatness conditions are

$$
\left(\Lambda(\phi)^{\prime} / \Lambda(\phi)\right)^{2} / 2=8 \phi^{6} /\left(M^{4}+\phi^{4}\right)^{2}<<1
$$

and

$$
\Lambda(\phi)^{\prime \prime} / \Lambda(\phi)=12 \phi^{2} /\left(M^{4}+\phi^{4}\right)<<1
$$

Generally, for a controllable theory it is necessary that the effective potential only valid at scales lower than Planck energy scale.

We can have this quartic term potential from a simple superpotential, Wess-Zumino mode12,

$$
W=c \phi^{3}
$$

(where $\mathrm{c}$ is a self-coulping constant) with the following $\mathrm{U}(1) \mathrm{R}$-symmetry

$$
\phi \rightarrow \exp (i \beta / 3) \phi
$$

where $\beta$ is the transformation parameter and

$$
W \rightarrow \exp (i \beta) W
$$

plusing a cosmological constant-like energy term. Thus, this symmetry forbiddes the other higher order terms in $\phi$ and the resultant potential possesses a $Z_{4}$ symmetry. Generally, if we require the system having a $Z_{2}$ symmetry the potential should also include the $\phi^{2}$ term 13.15 .20, that is the mass term.

As the treatment in literature 16 we define a parameter $z=r / m$, and another one, the " charge-mass-ratio" parameter $\alpha=q / m$ with $m>0$ as well as $r>0$ and

$$
y=3\left(z^{2}-2 z+\alpha^{2}\right) / z^{4}
$$

It is easy to find when $a(t, r)=0$

$$
\Lambda m^{2}=y
$$

Generally equation $(2)$ when $\mathrm{a}(\mathrm{t}, \mathrm{r})=0$ possesses four un-degenerate solutions and equation

$$
d y / d z=0
$$

has two, among which in our case, at the moment, only the small value one is relevent to our following analysis, that is

$$
0<\alpha^{2}<9 / 8
$$


and

$$
z_{-}=3 / 2-\left(9 / 4-2 \alpha^{2}\right)^{1 / 2} .
$$

We have the following observations for the charged de Sitter black hole spacetimes that there shall exist that (we take $\Lambda$ as the value of the potential)

a. Two horizons provided $\Lambda m^{2}>y\left(z_{-}\right)$,

b. One horizon if $\Lambda m^{2}=y\left(z_{-}\right)$and

c. No horizon when $\Lambda m^{2}<y\left(z_{-}\right)$

The family of parameter, say, $S[q, m, M, b]$, such that for the value of $\Lambda$ not less than certain value $y\left(z_{-}\right) / m^{2}$, black holes are formed, otherwise no black holes are formed.

Then, (b), the critical solution is universal with respect to the family of initial data considered,

$$
b\left(M^{4}+\phi^{4}\right) m^{2}=y\left(z_{-}\right)
$$

and

$$
y\left(z_{-}\right)=3 \frac{\left[\left(3-\sqrt{9-8 \alpha^{2}}\right)^{2} / 4-3+\left(9-8 \alpha^{2}\right)^{1 / 2}+\alpha^{2}\right]}{\left[3-\left(9-8 \alpha^{2}\right)^{1 / 2}\right]^{4}}
$$

That is, the right hand side of Eq.(15) is only a function of the "charge-massratio"

$$
b\left(M^{4}+\phi^{4}\right) m^{2}=f(\alpha)
$$

In the standard scenario of inflation the inflating expansion lasts about a Planck time with some 50 e-foldings to solve mainly the original monopole, geometric flatness and physical horizon problem 3 . After that the inflaton will execute oscillations around the minimum of the inflation potential, to convert its stored energy into the to be created physical world during the reheating period 10. In the charged de Sitter black hole case as we discuss the inflaton energy transfered to form a black hole is constrained by the black hole's charge-mass-ratio $\alpha$ mathematically, which decides the energy transfering rate to form black holes. Of cause, the details of energy transfer mechanism need more physics inputs and theoretical considerations, especially that where the charges come from specifically, besides some proposals such as in $\mathrm{PBHs}$ pair production or parameter resonance in preheating erd if the inflaton coupling with other boson or charged fermion fields. It is similar and straightway to discuss black holes in de Sitter spacetime without charge, which will be discussed following by taking $q=0$. In this case the parameter set only consists of three elements $S[m, M, b]$ and there still shall exist that, with differences from the charged case,

a. No horizon provided $3 \Lambda>1 / m$ (the root is negative), 
b. One degenerate horizon from three horizons if $3 \Lambda=1 / m$ with horizon at $r=1 / \Lambda^{1 / 2}$, that is, the horizon increases with energy input, which is obvious in the simplest Schwarzchild metric case and the energy transfering in the uncharged case satisfies

$$
b\left(M^{4}+\phi^{4}\right) m=1 / 3
$$

c. Two distinct horizons when $3 \Lambda<1 / m$ with horizons at $r_{1}=2 \cos (\delta) / \Lambda^{1 / 2}$ and $r_{2}=2 \cos (\delta / 3+4 \pi / 3) / \Lambda^{1 / 2}$ respectively, under condition $\cos (\delta)=-3 m \Lambda^{1 / 2}$. And the third root of solutions turns out being negative.

In this simpler case it is clear to see that there still exists the similar energy transfering relation Eq.(11), but without additional parameter constraint as the charged case and the classical thermodynamics quantities to be calculated are dependent on the slow changing inflaton, which is very interesting. We will detail the tedious computations and publish the results elsewher 19.

The cosmological term form chosen as the one given by Peeples and Vilenkin is due to the following two reasons. One is the connection to the tree level hybrid inflation model in the very early Universe era, which is a very promising theory to confront all astrophysics observations as we have known so fa 15.1 , 20.6. Another is from the convincing and consistent results of recent analyses to the gravitational lensings, SNe Ia as well as large-scale structure observation 11 and theoretical physics considerations with inconsistence predictions from theories 9 that disfavor classes of quintessence models or a simplest cosmological constant interpretation.

\section{Acknowledgements}

Xin He Meng is indebted to valuable discussions on this topic with Laura Covi, Robert Brandenberger, Ilia Gogoladze, Christopher Kolda, David Lyth, Leszek Roszkowski, Lewis Ryder, Goran Senjanovic, R.Tung and Xinmin Zhang during his stay at Lancaster University, UK and ICTP, Italy. He also thanks Profs S.Randjbar-Daemi, Goran Senjanovic and A.Smirnov for kind invitation for one month visit at ICTP. The authors would all like to express their gratitude to Abdus Salam ICTP for hospitality extended to them during this work being completed. This work is partly supported by grants from National Education Ministry and Natural Science foundation of P.R.China

References

1. M.Turner and J.Tyson, astro-ph/9901113 and references therein 
2. M.Turner, astro-ph/9901168 and references therein, Physica Scripta, in press astro-ph/9901109); L.Wang, R.Caldwell, J.Ostriker and P.Steinhardt, astro-ph/9901388.

3. A.Linde, Particle Physics and Inflationary Cosmology, Harwood Academic, Switzerland(1990).

4. R.Bousso and S.Hawking, Phys.Rev.D57,2436(1998); ibid 28,6312(1996), qr-qc/9608009

5. Y.Zeldovich and I.Novikov, Sov.Astron.A.J.10,602(1967); S.Hawking, MNRAS 152,75(1971); A.R. Liddle and A.Green, astro-ph/9710235

6. L.Kofman, astro-ph/9605155 in Relativistic Astrophysics: in Honor of Igor Novikov's 60th Birthday; L.Kofman,A.Linde and A.Starobinsky, Phys.Rev.D56,3258(1997)

7. A.R.Liddle and D.Lyth, Cosmological Inflation and Large Scale Structure,to be published by Oxford University Press, Phys.Rep.231,1(1993); E.Kolb and M.Turner, The early Universe, Addison-wesley(1990)

8. P.Steinhardt and M.Turner,Phys.Rev.D29,2162(1984); J.Garcia-Bellido and A.Linde, hep-th/9408023 and references therein

9. S.Weinberg,Rev.Mod.Phys.61,1(1989); C.Kolda and D.Lyth, hep$\mathrm{ph} / 9811375$,

10. V.Zanchin, et al, hep-ph/9901207 and references therein

11. A. Cooray and D. Huterer, astro-ph/9901097; N. Bahcall, astroph/9901076 and reference therein; S. Perlmutter, M. Turner and M.White, astro-ph/9901052.

12. J.Wess and J.Bagger,Supersymmetry and Supergravity, (Princeton University Press, Princeton 1983)

13. M.Dine and A.Riotto, Phys.Rev.Lett.79,2632(1997); X.H.Meng, hepph/9809614; H.Nilles, Phys.Rep.110,1(1984).

14. M.Turner, astro-ph/9811454 and references therein

15. D.H.Lyth and A.Riotto, hep-ph/9807278 and Phys.Rep. to appear and references therein

16. K.Lake and R.Roeder, Phys.Rev.D15, 3513(1977); K.Lake, ibid. 19, 421(1979)

17. M.W. Choptuik, Phys. Rev. Lett. 70, 9 (1993); A.M.Green and A.R.Liddle, Phys.Rev.D54,6166(1997), astro-ph/9901268

18. P.Peebles and A.Vilenkin, astro-ph/9810509

19. X.H.Meng, et al, to appear

20. M.Dine, hep-ph/9612389 and references therein 